\title{
Fragmentation Pathways of Trifluoroacetyl Derivatives of Methamphetamine, Amphetamine, and Methylenedioxyphenylalkylamine Designer Drugs by Gas Chromatography/Mass Spectrometry
}

\author{
Takeshi Kumazawa, ${ }^{1}$ Kenji Hara, ${ }^{2}$ Chika Hasegawa, ${ }^{3}$ Seisaku Uchigasaki, ${ }^{4}$ \\ Xiao-Pen Lee, ${ }^{1}$ Hiroshi Seno, ${ }^{5}$ Osamu Suzuki, ${ }^{6}$ and Keizo Sato ${ }^{1}$ \\ ${ }^{1}$ Department of Legal Medicine, Showa University School of Medicine, 1-5-8 Hatanodai, Shinagawa-ku, Tokyo 142-8555, Japan \\ ${ }^{2}$ Department of Forensic Medicine, Fukuoka University School of Medicine, 7-45-1 Nanakuma, Jonan-ku, Fukuoka 814-0180, Japan \\ ${ }^{3}$ Department of Legal Medicine, Toho University School of Medicine, 5-21-16 Omorinishi, Ota-ku, Tokyo 143-8540, Japan \\ ${ }^{4}$ Department of Legal Medicine, Nihon University School of Medicine, 30-1 Ohyaguchi-kamicho, Itabashi-ku, Tokyo 173-8610, Japan \\ ${ }^{5}$ Department of Legal Medicine, Aichi Medical University School of Medicine, Nagakute-cho, Aichi 480-1195, Japan \\ ${ }^{6}$ Department of Legal Medicine, Hamamatsu University School of Medicine, 1-20-1 Handayama, Higashi-ku, \\ Hamamatsu 431-3192, Japan
}

Correspondence should be addressed to Takeshi Kumazawa, kumazawa@med.showa-u.ac.jp

Received 24 February 2011; Accepted 26 June 2011

Academic Editor: Stefan Schmatz

Copyright (C) 2011 Takeshi Kumazawa et al. This is an open access article distributed under the Creative Commons Attribution License, which permits unrestricted use, distribution, and reproduction in any medium, provided the original work is properly cited.

\begin{abstract}
Methamphetamine (MA), amphetamine (AM), and the methylenedioxyphenylalkylamine designer drugs, such as 3,4-methylenedioxymethamphetamine (MDMA), 3,4-methylenedioxyethylamphetamine (MDEA), N-methyl-1-(3,4-methylenedioxyphenyl)2-butanamine (MBDB), 3,4-methylenedioxyamphetamine (MDA), and 3,4-(methylenedioxyphenyl)-2-butanamine (BDB), are widely abused as psychedelics. In this paper, these compounds were derivatized with trifluoroacetic (TFA) anhydride and analyzed by gas chromatography/mass spectrometry using electron ionization in positive mode. Gas chromatographic separation for TFA derivatives of all compounds was successfully resolved using an Equity- 5 fused silica capillary column with a poly (5\% diphenyl95\% dimethylsiloxane) stationary phase. Base peaks or prominent peaks of MA, AM, MDMA, MDEA, MBDB, MDA, and BDB appeared at $m / z 154,140,154,168,168,135$, and 135 , respectively. These occurred due to $\alpha$-cleavage from the amide nitrogen, splitting into the TFA imine species and benzyl or methylenedioxybenzyl cations. Further prominent fragment ions at $m / z 118$ for MA and AM, $m / z 162$ for MDMA, MDEA, and MDA, and $m / z 176$ for MBDB and BDB were produced by cleavage of the phenylpropane or methylenedioxypropane hydrocarbon radical cation via a hydrogen rearrangement. These fragmentation pathways for the TFA derivatives of all the compounds are summarized and illustrated in this paper.
\end{abstract}

\section{Introduction}

In recent years, extensive attention in clinical and forensic toxicology has focused on the increasing abuse of methamphetamine (MA), amphetamine (AM), and methylenedioxyphenylalkylamine derivatives, such as 3,4-methylenedioxymethamphetamine (MDMA), 3,4-methylenedioxyethylamphetamine (MDEA), N-methyl-1-(3,4-methylenedioxyphenyl) -2-butanamine (MBDB), 3,4-methylenedioxyamphetamine (MDA), and 3,4-(methylenedioxyphenyl)-2-butanamine
(BDB). A number of severe and even fatal intoxications attributable to these drugs have been reported [1-4]. Consequently, detection and identification analyses for these compounds are routinely performed in clinical and forensic laboratories.

Several gas chromatographic methods to analyze MA, AM, MDMA, MDEA, MBDB, MDA, and BDB in doping control and toxicological analysis have been reported [58 ]. Because of their relatively low molecular weights, high 


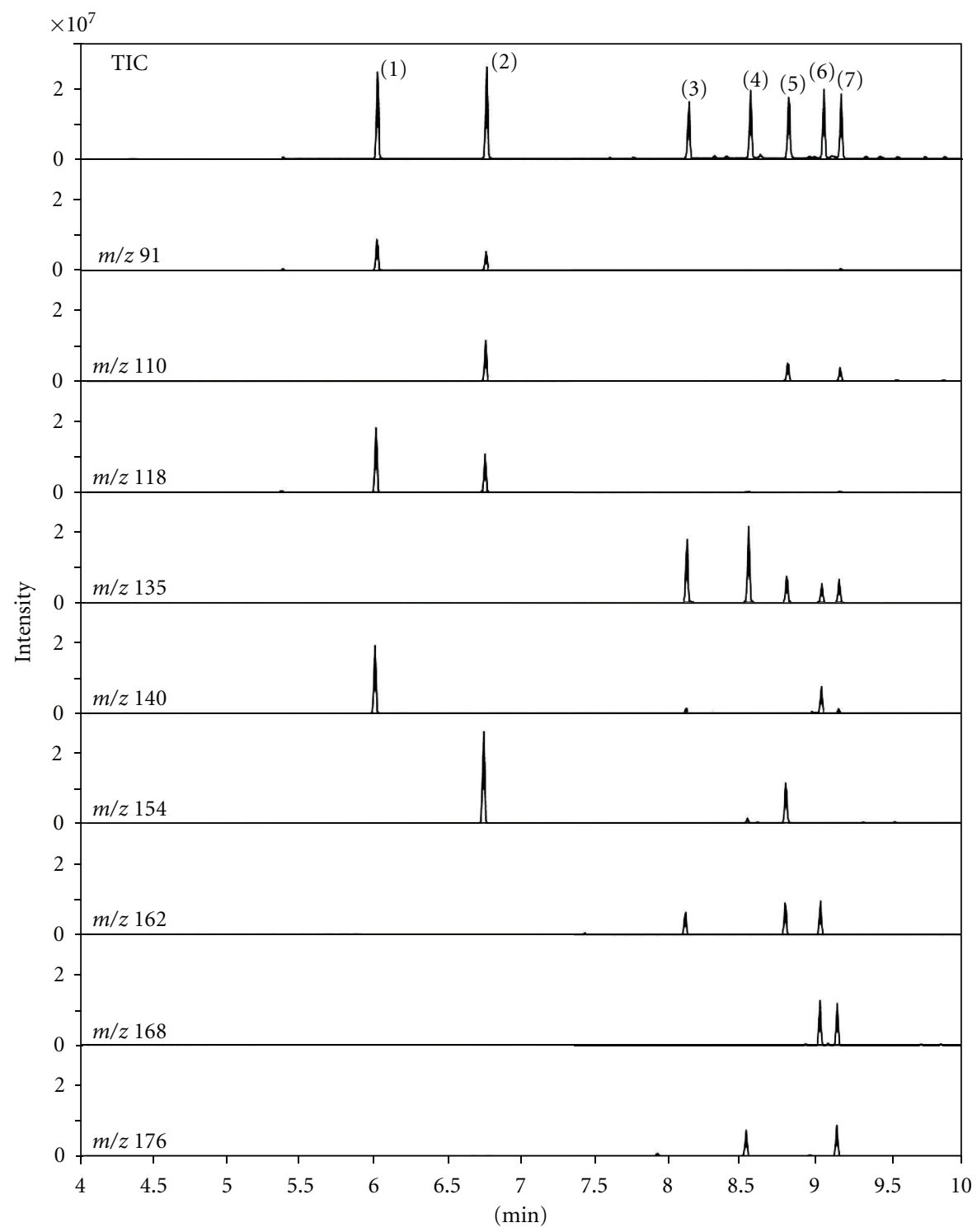

Figure 1: Mass chromatograms obtained from GC/MS analysis using Equity-5 capillary column for TFA derivatives of seven analytes. The ionizing energy was $70 \mathrm{eV}$ with an emission current of $60 \mu \mathrm{A}$. Ten nanograms of each analyte were injected in the positive-ion EI mode. Peaks: (1) AM-TFA; (2) MA-TFA; (3) MDA-TFA; (4) BDB-TFA; (5) MDMA-TFA; (6) MDEA-TFA; and (7) MBDB-TFA.

polarity, and volatility, derivatization is necessary when using gas chromatography (GC) [9]. Acylation is one of the most popular derivatization reactions for primary and secondary amines and converts compounds into derivatives that are more easily separated or give an enhanced response in GC compared with the parent compound [10]. GC/mass spectrometry (MS) using the electron ionization (EI) mode is a widely used technique in drug analysis, as it leads to a number of fragment ions providing structural information [11]. Although quantitative analysis of MA, AM, and the methylenedioxyphenylalkylamine designer drugs has been frequently performed in clinical and forensic toxicology by GC/MS-EI with derivatization [8, 12-17], systematic studies of mass spectrometric behavior for these compounds have been limited $[9,18,19]$. In this paper, we present mass spectra and detailed fragmentation pathways for MA, AM,
MDMA, MDEA, MBDB, MDA, and BDB using GC/MS in EI mode after acylation derivatization.

\section{Experimental Part}

2.1. Materials. Hydrochloride salts of MDA, BDB, MDMA, and $\mathrm{MBDB}$ were prepared as described briefly here. MDA and $\mathrm{BDB}$ syntheses were performed according to the procedures described by Lindeke and Cho [20]. MDA was synthesized by hydrogenation of 1-(3,4-methylenedioxyphenyl)-2nitropropene, which was prepared beforehand by condensation of piperonal and nitroethane. BDB was synthesized by hydrogenation of 1-(3,4-methylenephenyl)-2-nitrobutene, which was prepared beforehand by condensation of piperonal and nitropropane. MDMA and MBDB syntheses were performed according to the procedures described by 

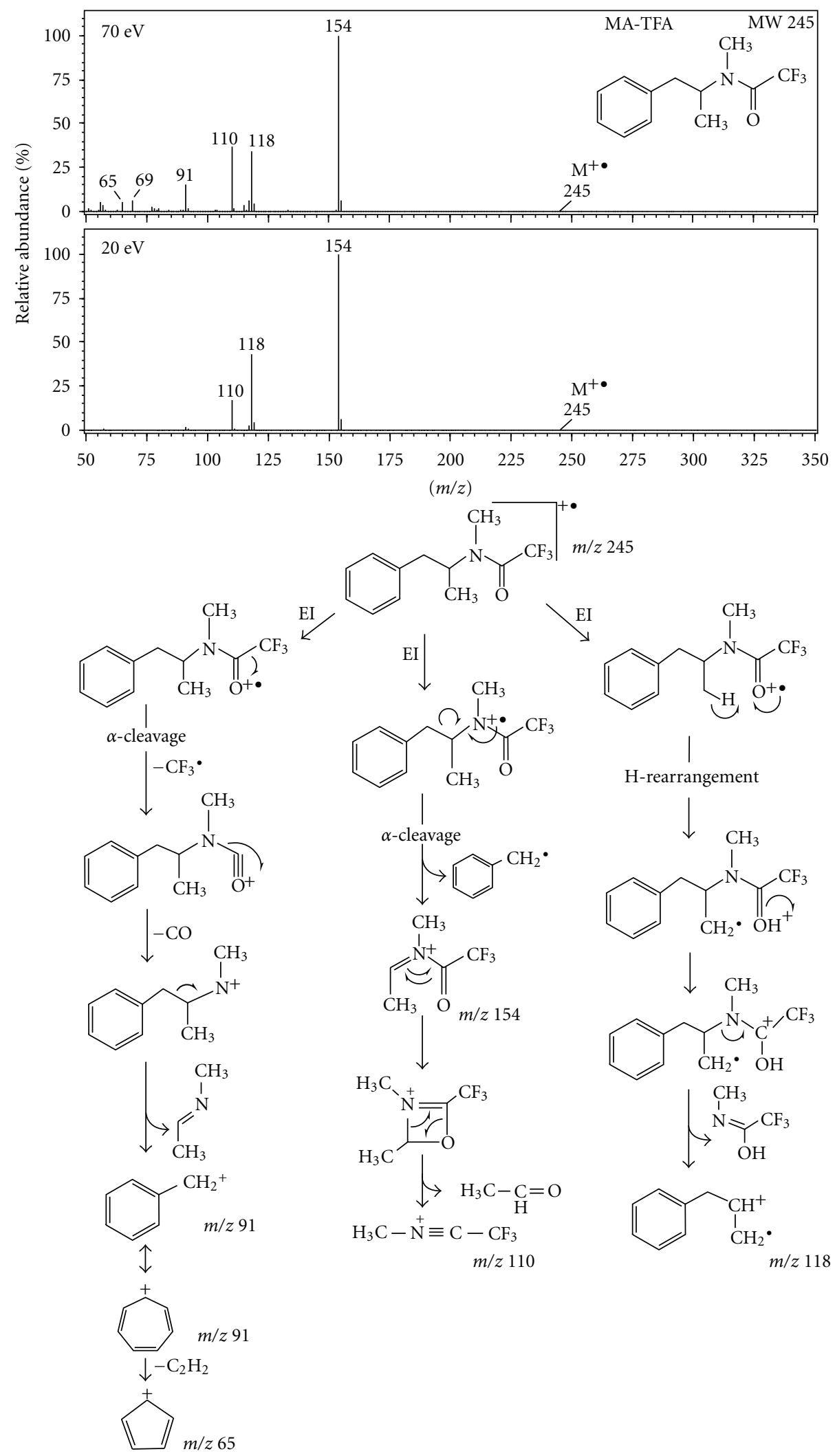

FIGURE 2: EI mass spectra of the TFA derivative of MA and their probable fragmentation pathway. 


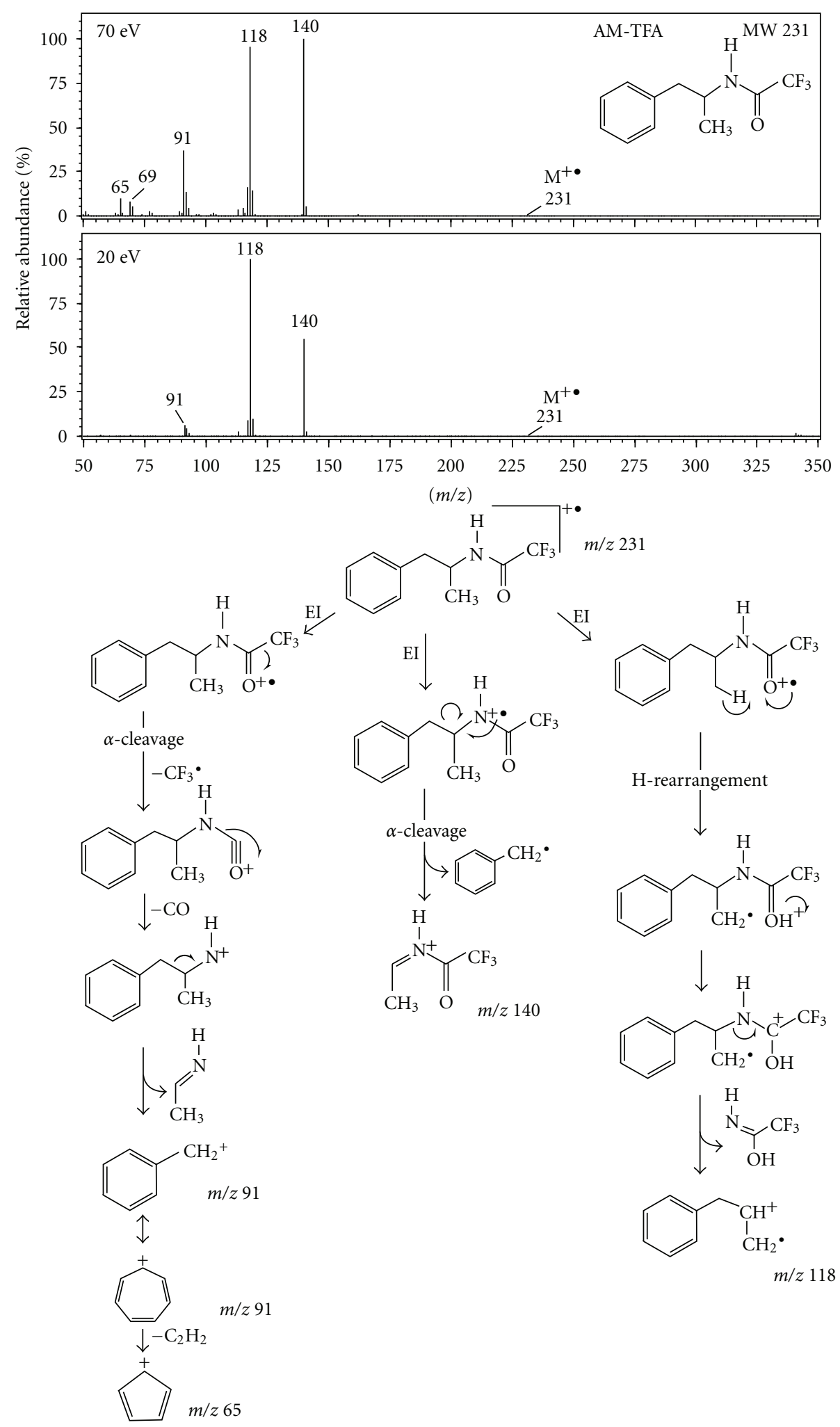

FIGURE 3: EI mass spectra of the TFA derivative of AM and their probable fragmentation pathway. 

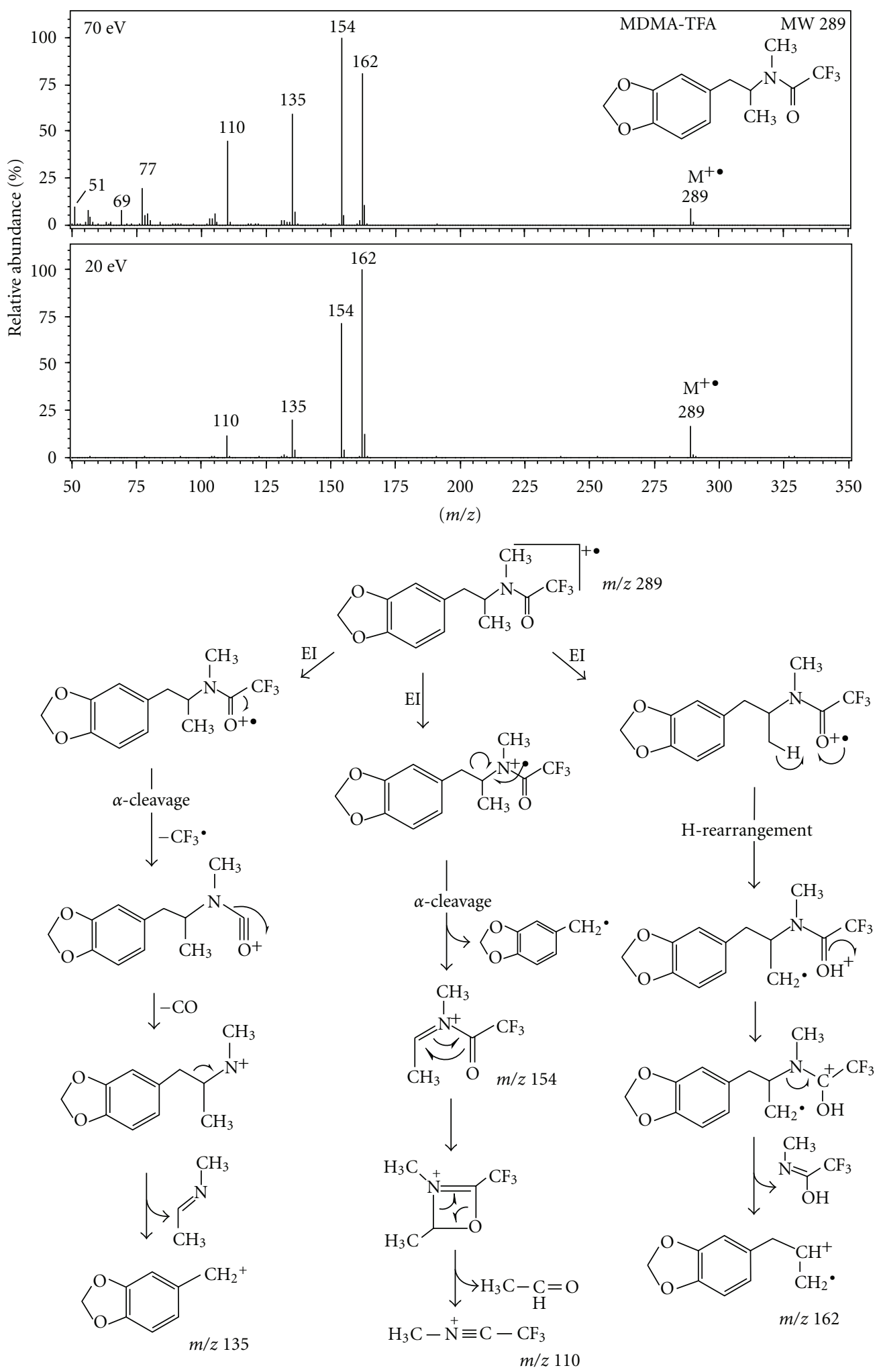

FIGURE 4: EI mass spectra of the TFA derivative of MDMA and their probable fragmentation pathway. 

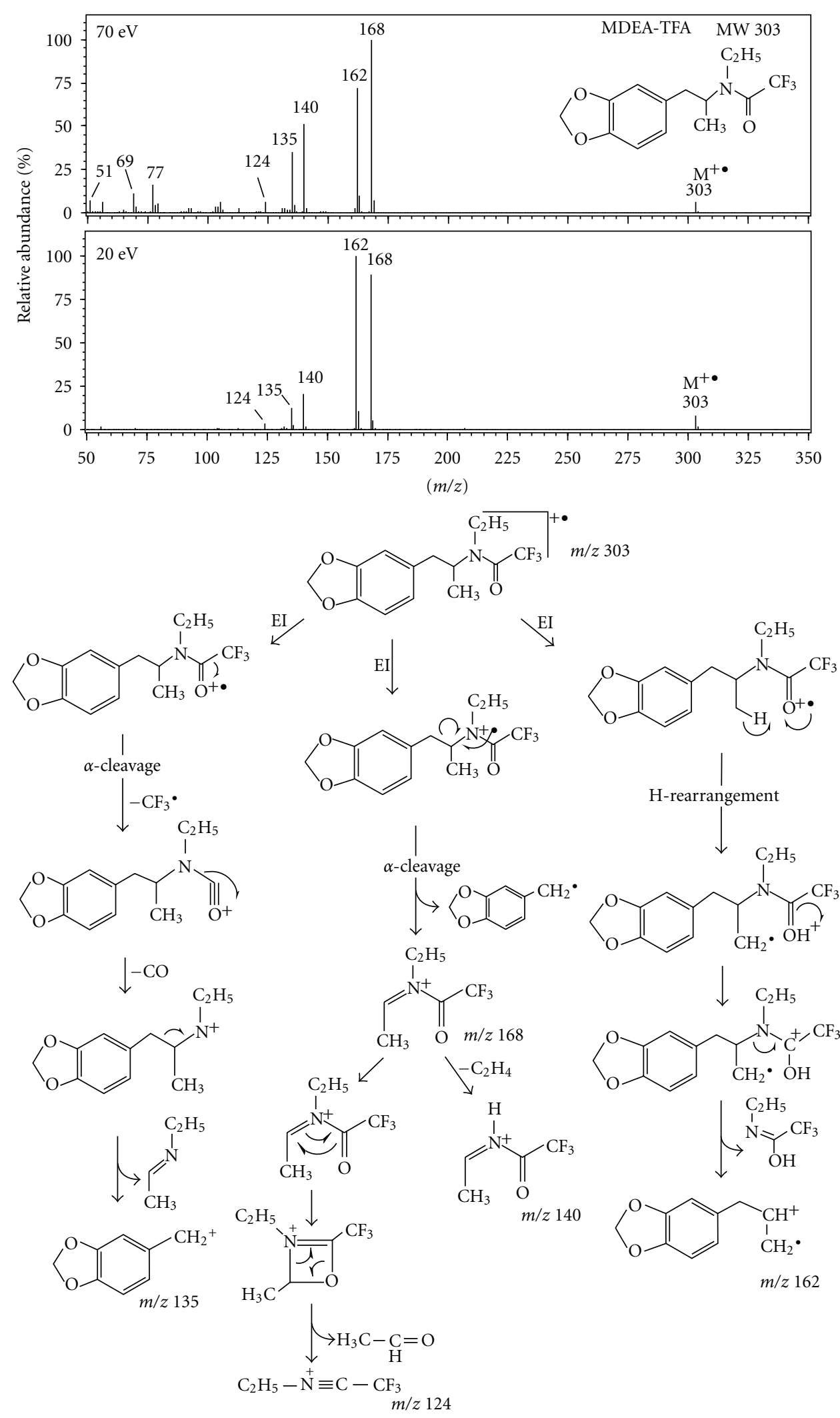

FIGURE 5: EI mass spectra of the TFA derivative of MDEA and their probable fragmentation pathway. 

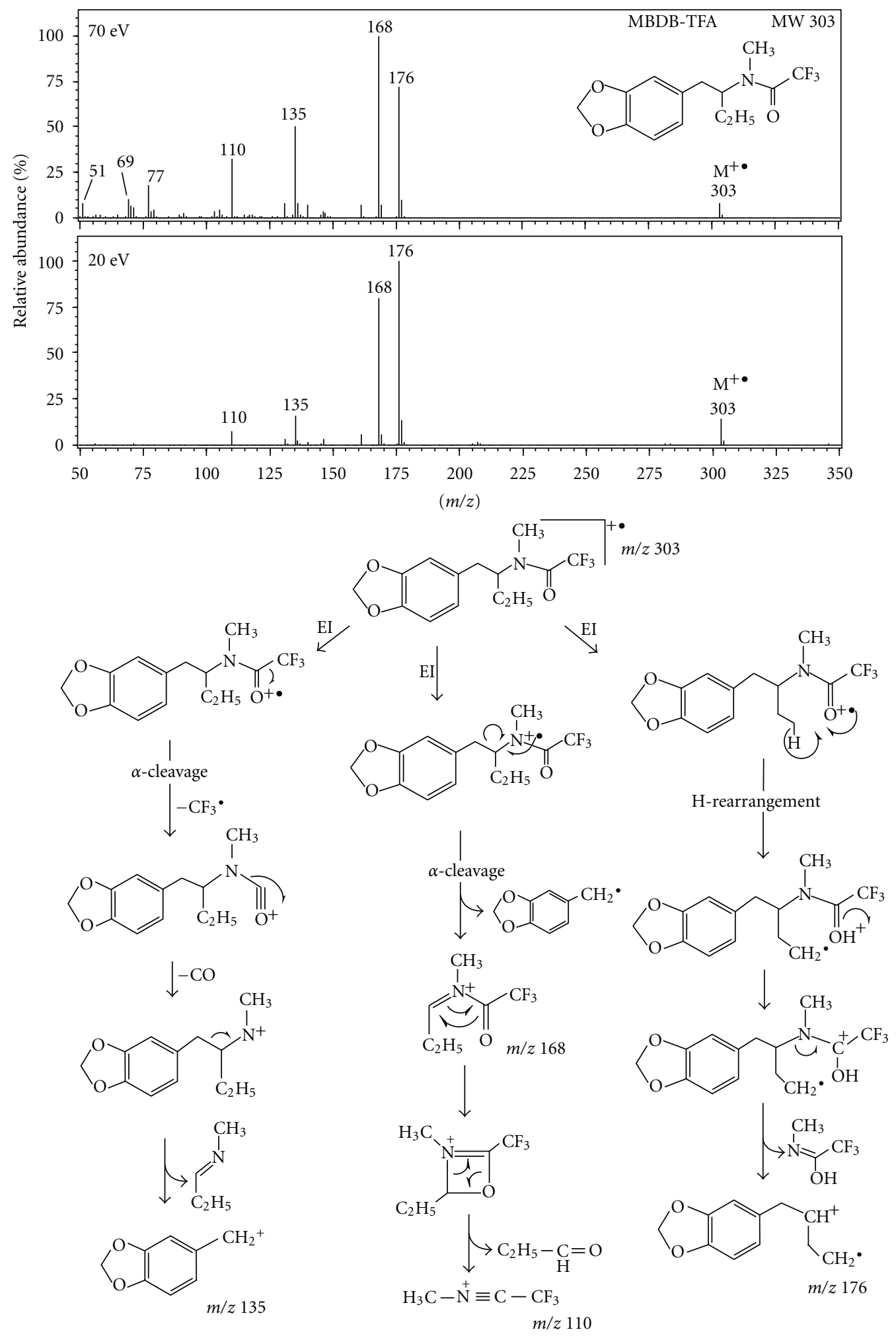

FIGURE 6: EI mass spectra of the TFA derivative of MBDB and their probable fragmentation pathway. 

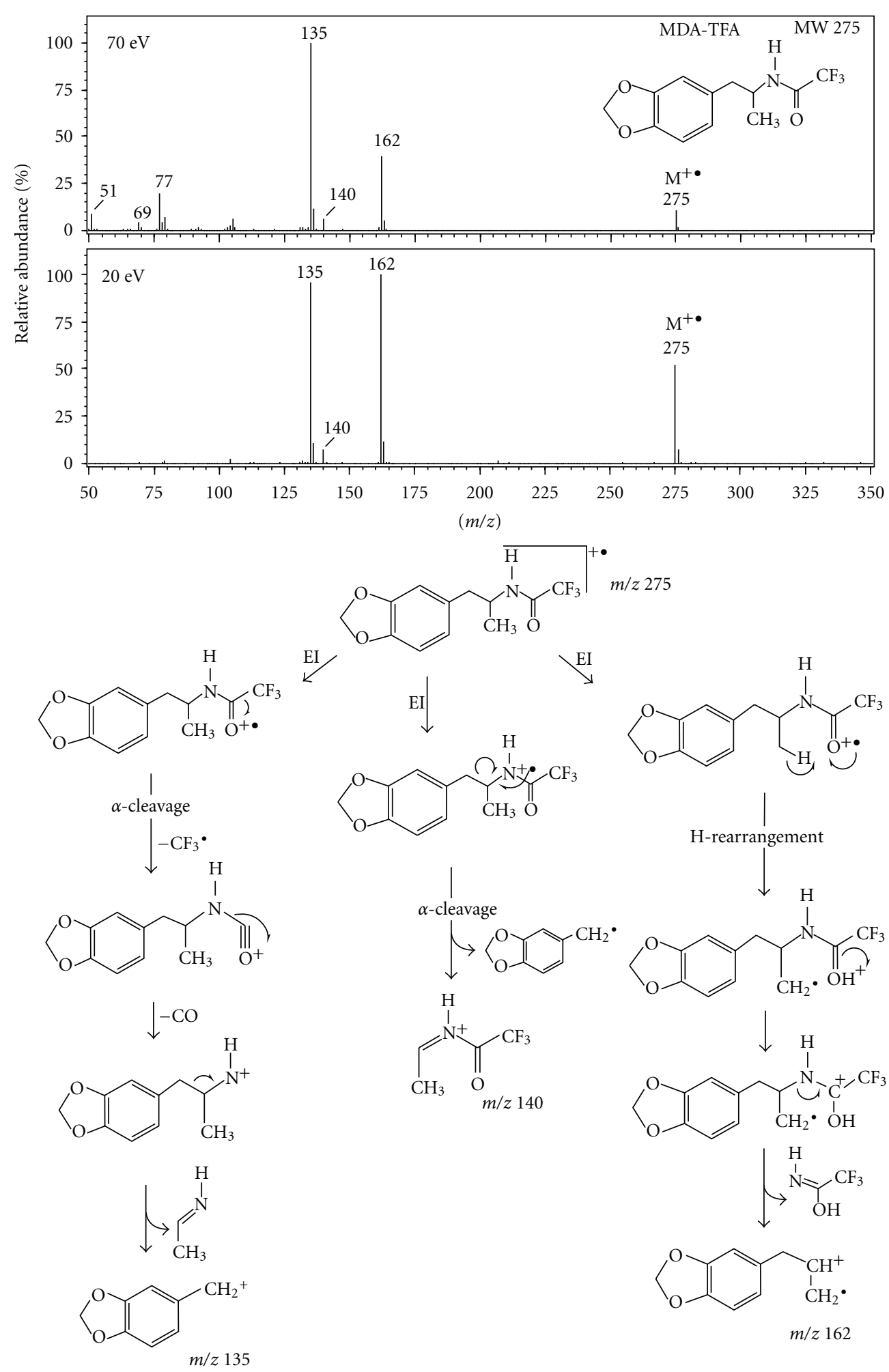

FIgURE 7: EI mass spectra of the TFA derivative of MDA and their probable fragmentation pathway.

Repke et al. [21]. MDMA and MBDB were prepared by hydrogenation of MDA and $\mathrm{BDB}$, respectively, followed by benzyloxycarbonylation with benzyloxycarbonyl chloride. These four compounds, MDA, BDB, MDMA, and MBDB, were then finally converted to their hydrochloride salts.
MDEA hydrochloride was synthesized according to published procedures [22] that follow. Briefly, acetic anhydride was added to a solution of MDA free base in pyridine and the mixture was stirred at room temperature for $0.5 \mathrm{~h}$. The reaction was quenched by addition of distilled water and 

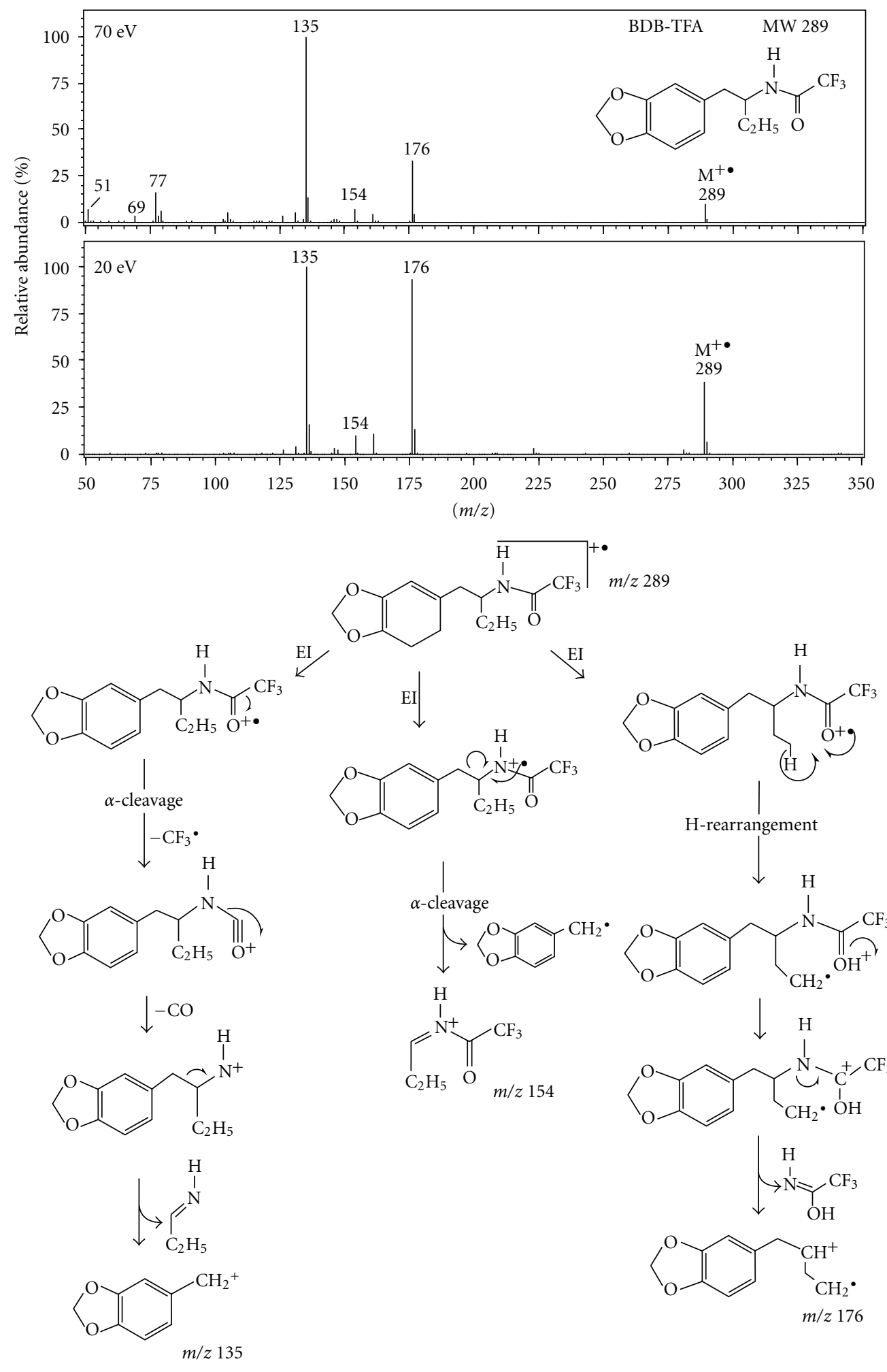

FIGURE 8: EI mass spectra of the TFA derivative of BDB and their probable fragmentation pathway.

acidified with hydrochloric acid. The aqueous mixture was extracted with diethyl ether and the organic phase was evaporated to dryness in vacuo. The residue, $\mathrm{N}$-acetyl-3,4methylenedioxyamphetamine, was recrystallized from ethyl acetate/hexane, and the crystalline product was added to a solution of acetic anhydride and pyridine. The resulting solution of $\mathrm{N}$-acetyl-3,4-methylenedioxyamphetamine was added to lithium aluminum hydride in anhydrous tetrahydrofuran and the reaction mixture was heated at reflux for three days. After cooling the reaction mixture on an ice-bath, 
the excess hydride was decomposed by addition of distilled water then sodium hydroxide. The mixture was filtered and the solvent removed in vacuo. The residue was dissolved in ethyl alcohol and concentrated hydrochloric acid was added. This aqueous solution was extracted with ethanol/diethyl ether followed by diethyl ether, and the solvent removed in vacuo. Recrystallization of the residue gave MDEA hydrochloride.

AM sulfate was synthesized according to the literature procedure [20]. All the compounds described above were prepared at the Department of Forensic Medicine, Fukuoka University School of Medicine. The salts were pure and characterized by mass spectrometry. MA hydrochloride was purchased from Dainippon Pharmaceutical Co., Ltd. (Osaka, Japan). Trifluoroacetic (TFA) anhydride was obtained from Pierce (Rockford, Ill, USA). Other chemicals used were of the highest purity commercially available.

2.2. Preparation of Standard Solutions. Stock standard solutions of MA, AM, MDMA, MDEA, MBDB, MDA, and BDB were prepared separately by dissolving an accurately weighed amount of each compound in methanol to achieve a concentration of $1 \mathrm{mg} \mathrm{mL}^{-1}$. All stock solutions were stored at $4^{\circ} \mathrm{C}$. Working standard solutions from $1-5 \mu \mathrm{g} 10 \mu \mathrm{L}^{-1}$ in methanol were prepared by serial dilution from the stock standard solutions. Ten microliter working standard solutions were evaporated to dryness under a gentle stream of nitrogen and the residue was used for derivatization.

2.3. Derivatization. MA, AM, MDMA, MDEA, MBDB, MDA, and BDB were derivatized with TFA anhydride. A $100 \mu \mathrm{L}$ aliquot of TFA anhydride/ethyl acetate $(5: 1, \mathrm{v} / \mathrm{v})$ was added to each residue, and samples were capped, mixed, and heated at $80^{\circ} \mathrm{C}$ for $10 \mathrm{~min}$ with an aluminum block heater (Reacti-Therm Heating/Stirring Model; Pierce). After cooling to room temperature, the solvent was then evaporated to dryness under a stream of nitrogen, and residues were reconstituted in $50 \mu \mathrm{L}$ ethyl acetate. A $1 \mu \mathrm{L}$ aliquot of sample solution was submitted for GC/MS analysis.

2.4. GC/MS Conditions. All analyses were performed using a Shimadzu GC-2010 gas chromatograph interfaced with a Shimadzu QP-2010 quadrupole mass spectrometer (Shimadzu Corp., Kyoto, Japan). The GC/MS was operated with an interface temperature of $300^{\circ} \mathrm{C}$ and an ionization source temperature of $250^{\circ} \mathrm{C}$. The mass spectrometer was tuned daily, using perfluorotributylamine. A solvent delay of $4.0 \mathrm{~min}$ was set to protect the filament from oxidation. Chromatographic separation was achieved using an Equity-5 fused silica capillary column $(30 \mathrm{~m} \times 0.32 \mathrm{~mm}$ i.d., $0.25 \mu \mathrm{m}$ film thickness, poly(5\% diphenyl-95\% dimethylsiloxane) stationary phase; Supelco, Bellefonte, Pa, USA). Helium, with a minimum purity of $99.99995 \%$, was used as carrier gas at a constant pressure of $42.3 \mathrm{kPa}$ (initial flow rate of $2 \mathrm{~mL} \mathrm{~min}^{-1}$ ). The gas chromatograph was equipped with a split/splitless injection port, operated at $250^{\circ} \mathrm{C}$. Samples were injected in the splitless mode, at a column temperature of $60^{\circ} \mathrm{C}$, and the splitter was then opened after $1 \mathrm{~min}$. The gas chromatograph oven temperature was programmed as follows: initial temperature, $60^{\circ} \mathrm{C}$ for $1 \mathrm{~min}$; from 60 to $200^{\circ} \mathrm{C}$ at $20^{\circ} \mathrm{C} \mathrm{min}^{-1}$; finally from 200 to $300^{\circ} \mathrm{C}$ at

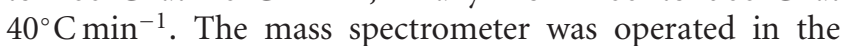
positive-ion EI mode using ionizing energy of $70 \mathrm{eV}$ and emission current of $60 \mu \mathrm{A}$, or ionizing energy of $20 \mathrm{eV}$ and emission current of $10 \mu \mathrm{A}$. Full-scan data were obtained with mass range of $m / z 50-350$, scan interval of $0.5 \mathrm{~s}$, and scan speed of $769 \mathrm{amu} / \mathrm{s}$.

\section{Results and Discussion}

TFA anhydride is the most widely used derivatizing agent, known to react with and acylate the primary and secondary amine groups of the amphetamine-type illicit drugs $[8,10$, 12, 15-17, 23]. However, excess TFA and byproducts such as, trifluoroacetic acid, are produced in reactions with the target compounds [24]. These have to be removed from the extract prior to the GC/MS analysis, in order to avoid damaging to the GC column [25]. In the present study, our sample preparation of drying the reaction mixture with TFA anhydride under a stream of nitrogen and reconstituting the residue in ethyl acetate greatly reduced both excess derivatizing agent and the acid byproduct. The TFA derivatives of the seven compounds were well separated with good peak shapes and no remarkable impurities within $9.5 \mathrm{~min}$ (Figure 1). In the preliminary experiment, the mixtures of MA, AM, MDMA, MDEA, MBDB, MDA, and BDB were compared on different stationary phases using several temperature programs. The best compromise between analysis time and resolution was achieved on the Equity- 5 capillary column.

Figures 2-8 show the EI full-scan mass spectra with ionizing energies of $70 \mathrm{eV}$ and $20 \mathrm{eV}$ for the TFA derivatives of MA, AM, MDMA, MDEA, MBDB, MDA, and BDB and their probable fragmentation pathways. The molecular ions for MA and AM were barely detectable and therefore of little quantitative value, at $\mathrm{m} / z 245$ and $\mathrm{m} / z$ 231, respectively, (Figures 2 and 3). MDMA, MDEA, MBDB, MDA, and BDB produced molecular ions with relatively high abundance (6$12 \%$ at $70 \mathrm{eV}$ and $9-53 \%$ at $20 \mathrm{eV}$ ) at $m / z 289,303,303,275$, and 289 , respectively (Figures $4-8$ ). The relative abundance of molecular ions in the EI mass spectra of these derivatives may depend substantially on the chemical nature of substituents directly attached to the benzene ring, such as their inductive effect and/or their thermal stability.

MA and AM produced prominent peaks at $\mathrm{m} / \mathrm{z} 154$ and 140 , respectively, in the mass spectra (Figures 2 and 3 ). These ions were the TFA imine species, probably by $\alpha$-cleavage of the amide nitrogen of their parent molecules. This cleavage could also simultaneously lead to benzyl cation $\left(\left[\mathrm{C}_{7} \mathrm{H}_{7}\right]^{+}\right)$ fragment at $m / z$ 91. The mass spectra for both derivatives showed high relative abundance ions at $m / z 118(35-44 \%$ for MA and $97-100 \%$ for AM), corresponding to the phenylpropane hydrocarbon radical cation (Figures 2 and 3 ). The formation of this cation can be explained by a hydrogen rearrangement [26]. This involves migration of a $\gamma$-hydrogen atom from the alkyl group to the carbonyl oxygen through a cyclic six-membered transition state, followed by cleavage of the alkyl carbon-nitrogen bond in the side chain leading to the loss of imine species. 
For MDMA, MDEA, and MBDB, prominent peaks at $\mathrm{m} / \mathrm{z}$ 154 (for MDMA) and 168 (for MDEA and MBDB) and the 3,4-methylenedioxybenzyl cation peak at $\mathrm{m} / \mathrm{z} 135$ probably resulted from $\alpha$-cleavage of the amide nitrogen (Figures 4-6). MDA and BDB both have $\mathrm{H}$ as a substituent on the nitrogen atom and both gave base peak ions at $m / z 135$ corresponding to the 3,4-methylenedioxybenzyl cation (Figures 7 and 8), produced via $\alpha$-cleavage of the amide of their parent molecules. The spectra of these methylenedioxy derivatives showed characteristic fragment ions at $m / z 162$ for MDMA, MDEA, and MDA and $m / z 176$ for MBDB and BDB, with high relative abundance of $33-97 \%$ at $70 \mathrm{eV}$ and $94-100 \%$ at $20 \mathrm{eV}$. Both of these fragment ions can be assigned as the methylenedioxyphenylpropane radical cation due to a hydrogen rearrangement (Figures 4-8).

The compounds with a methyl substituent on the nitrogen atom, MA, MDMA, and MBDB, gave a characteristic cation $\left(\left[\mathrm{CH}_{3}-\mathrm{N} \equiv \mathrm{C}-\mathrm{CF}_{3}\right]^{+}\right.$) at $\mathrm{m} / z 110$ (Figures 2, 4, and 6). MDEA with an ethyl substituent on the nitrogen atom produced analogous cation at $\mathrm{m} / \mathrm{z} 124$ corresponding to $\left(\left[\mathrm{C}_{2} \mathrm{H}_{5}-\mathrm{N} \equiv \mathrm{C}-\mathrm{CF}_{3}\right]^{+}\right)$, with low relative abundance of $4 \%$ at $20 \mathrm{eV}$ and $7 \%$ at $70 \mathrm{eV}$ (Figure 5). We propose that this resulted from the decomposition reaction of the four-membered nitrogen-containing heterocyclic intermediates in the fragmentation process of the $\mathrm{m} / z 154$ or 168 cations (Figures 2, 4-6). In addition, the spectrum of MDEA showed a characteristic cation at $\mathrm{m} / \mathrm{z} 140$ with a relative abundance of $55 \%$ at $70 \mathrm{eV}$ and $21 \%$ at $20 \mathrm{eV}$ (Figure 5). This iminium ion probably originated from a rearrangement of the ethyl group of the $\mathrm{m} / z 168$ cation to lose ethylene $\left(\mathrm{C}_{2} \mathrm{H}_{4}\right)$. These results were consistent with the previous report [27].

Benzyl or tropylium cation at $\mathrm{m} / \mathrm{z} 91$ produced in MA and AM occurred due to a neutral loss of acetylene $\left(\mathrm{C}_{2} \mathrm{H}_{2}\right)$, which gave rise to the cyclopentadienyl ion $\left(\left[\mathrm{C}_{5} \mathrm{H}_{5}\right]^{+}\right)$at $\mathrm{m} / z$ 65 (Figures 2 and 3). Thus, $m / z$ 91/65 for MA and AM was typical fragment pairs of monosubstituted alkyl aromatics, although these ions are less favored in the mass spectra. The spectra for MDA and BDB showed complementary ions at $\mathrm{m} / z 140$ and $\mathrm{m} / \mathrm{z} 154$, respectively, with relative abundance of $7 \%$ at $70 \mathrm{eV}$ and $9-11 \%$ at $20 \mathrm{eV}$, corresponding to the TFA imine species. The ion of $\mathrm{m} / z 69\left(\left[\mathrm{CF}_{3}\right]^{+}\right)$with 4$14 \%$ relative abundance at $70 \mathrm{eV}$ was present in the TFA derivatives of all compounds.

\section{Conclusions}

The GC/MS-EI ionization mass spectra of TFA derivatives of $\mathrm{MA}, \mathrm{AM}$, and the methylenedioxyphenylalkylamines, MDMA, MDEA, MBDB, MDA, and BDB, were studied in positive mode. The main fragmentation pathways for all seven derivatives involved $\alpha$-cleavage and a hydrogen rearrangement. Both pathways gave characteristic ions, occurring at $m / z 154,118$, and 91 for MA; $m / z 140,118$, and 91 for AM; $m / z$ 162, 154, and 135 for MDMA; $m / z$ 168, 162, 140, and 135 for MDEA; $m / z$ 176, 168, and 135 for MBDB; $\mathrm{m} / \mathrm{z} 162,140$, and 135 for MDA; and $\mathrm{m} / \mathrm{z} 176,154$, and 135 for BDB. Additionally, MA, MDMA, and MBDB with a methyl substituent on the nitrogen atom had an intense ion at $\mathrm{m} / \mathrm{z} 110$ from the fragmentation process of the $\mathrm{m} / \mathrm{z}$
154 or $\mathrm{m} / \mathrm{z} 168$ prominent peak ions. These characteristic fragmentation patterns of TFA derivatives of MA, AM, and the methylenedioxyphenylalkylamine designer drugs will aid in the identification of these drugs from biological samples in clinical and forensic toxicology.

\section{Acknowledgment}

This study was supported in part by a Grant-in-Aid for scientific research (C) from the Japan Society for the Promotion of Science (JSPS).

\section{References}

[1] V. Fineschi, F. Centini, E. Mazzeo, and E. Turillazzi, "Adam (MDMA) and Eve (MDEA) misuse: an immunohistochemical study on three fatal cases," Forensic Science International, vol. 104, no. 1, pp. 65-74, 1999.

[2] N. Carter, G. N. Rutty, C. M. Milroy, and A. R. W. Forrest, "Deaths associated with MBDB misuse," International Journal of Legal Medicine, vol. 113, no. 3, pp. 168-170, 2000.

[3] R. García-Repetto, E. Moreno, T. Soriano, C. Jurado, M. P. Giménez, and M. Menéndez, "Tissue concentrations of MDMA and its metabolite MDA in three fatal cases of overdose," Forensic Science International, vol. 135, no. 2, pp. 110114, 2003.

[4] J. L. Pilgrim, D. Gerostamoulos, O. H. Drummer, and M. Bollmann, "Involvement of amphetamines in sudden and unexpected death," Journal of Forensic Sciences, vol. 54, no. 2, pp. 478-485, 2009.

[5] P. R. Paetsch, G. B. Baker, L. E. Caffaro, A. J. Greenshaw, G. A. Rauw, and R. T. Coutts, "Electron-capture gas chromatographic procedure for simultaneous determination of amphetamine and N-methylamphetamine," Journal of Chromatography, vol. 573, no. 2, pp. 313-317, 1992.

[6] P. Marquet, E. Lacassie, C. Battu, H. Faubert, and G. Lachâtre, "Simultaneous determination of amphetamine and its analogs in human whole blood by gas chromatography-mass spectrometry," Journal of Chromatography B, vol. 700, no. 1-2, pp. 77-82, 1997.

[7] F. T. Peters, S. Schaefer, R. F. Staack, T. Kraemer, and H. H. Maurer, "Screening for and validated quantification of amphetamines and of amphetamine- and piperazine-derived designer drugs in human blood plasma by gas chromatography/mass spectrometry," Journal of Mass Spectrometry, vol. 38, no. 6, pp. 659-676, 2003.

[8] F. Moriya, "Accumulation of intravenously administered methamphetamine in stomach contents," Forensic Toxicology, vol. 28 , no. 1, pp. 43-46, 2010.

[9] G. Frison, L. Tedeschi, D. Favretto, A. Reheman, and S. D. Ferrara, "Gas chromatography/mass spectrometry determination of amphetamine-related drugs and ephedrines in plasma, urine and hair samples after derivatization with 2,2,2-trichloroethyl chloroformate," Rapid Communications in Mass Spectrometry, vol. 19, no. 7, pp. 919-927, 2005.

[10] D. R. Knapp, Handbook of Analytical Derivatization Reactions, Wiley \& Sons, New York, NY, USA, 1979.

[11] J. T. Cody and R. L. Foltz, "GC/MS analysis of body fluids for drugs of abuse," in Forensic Applications of Mass Spectrometry, J. Yinon, Ed., pp. 2-59, CRC Press, Boca Raton, Fla, USA, 1995.

[12] W. Weinmann, M. Renz, S. Vogt, and S. Pollak, "Automated solid-phase extraction and two-step derivatisation for simultaneous analysis of basic illicit drugs in serum by GC/MS," 
International Journal of Legal Medicine, vol. 113, no. 4, pp. 229-235, 2000.

[13] M. Pujadas, S. Pichini, S. Poudevida et al., "Development and validation of a gas chromatography-mass spectrometry assay for hair analysis of amphetamine, methamphetamine and methylenedioxy derivatives," Journal of Chromatography B, vol. 798, no. 2, pp. 249-255, 2003.

[14] J. L. Villamor, A. M. Bermejo, P. Fernández, and M. J. Tabernero, "A new GC-MS method for the determination of five amphetamines in human hair," Journal of Analytical Toxicology, vol. 29, no. 2, pp. 135-139, 2005.

[15] C. Hasegawa, T. Kumazawa, X. P. Lee et al., "Pipette tip solid-phase extraction and gas chromatography-mass spectrometry for the determination of methamphetamine and amphetamine in human whole blood," Analytical and Bioanalytical Chemistry, vol. 389, no. 2, pp. 563-570, 2007.

[16] S. Lee, Y. Park, W. Yang et al., "Development of a reference material using methamphetamine abusers' hair samples for the determination of methamphetamine and amphetamine in hair," Journal of Chromatography B, vol. 865, no. 1-2, pp. 33 39, 2008.

[17] K. Kudo, T. Ishida, W. Hikiji et al., "Construction of calibration-locking databases for rapid and reliable drug screening by gas chromatography-mass spectrometry," Forensic Toxicology, vol. 27, no. 1, pp. 21-31, 2009.

[18] C. R. Clark, J. DeRuiter, A. K. Valaer, and F. T. Noggle, "GCMS analysis of acylated derivatives of methamphetamine and regioisomeric phenethylamines," Journal of Chromatographic Science, vol. 33, no. 9, pp. 485-492, 1995.

[19] C. R. Clark, J. DeRuiter, and F. T. Noggle, "Chromatographic and mass spectrometric methods for the differentiation of N-methyl-1-(3,4-methylenedioxyphenyl)-2-butanamine from regioisomeric derivatives," Journal of Chromatographic Science, vol. 34, no. 5, pp. 230-237, 1996.

[20] B. Lindeke and A. K. Cho, "Specifically deuterated 1-phenyl-1isopropylamines. Synthesis of deuterium $( \pm)$-amphetamine, ( \pm )- $\alpha$-methyltyramine," Acta Pharmaceutica Suecica, vol. 9, no. 4, pp. 363-372, 1972.

[21] D. B. Repke, D. K. Bates, and W. J. Ferguson, "Synthesis of dextroamphetamine sulfate and methamphetamine hydrochloride from D-phenylalanine," Journal of Pharmaceutical Sciences, vol. 67, no. 8, pp. 1167-1168, 1978.

[22] A. T. Shulgin and A. Shulgin, PiHKAL: A Chemical Love Story, Transform Press, Berkeley, Calif, USA, 1991.

[23] J. Y. Kim, K. S. Jung, M. K. Kim, J. I. Lee, and M. K. In, "Simultaneous determination of psychotropic phenylalkylamine derivatives in human hair by gas chromatography/ mass spectrometry," Rapid Communications in Mass Spectrometry, vol. 21, no. 11, pp. 1705-1720, 2007.

[24] C. Brede, I. Skjevrak, and H. Herikstad, "Determination of primary aromatic amines in water food simulant using solidphase analytical derivatization followed by gas chromatography coupled with mass spectrometry," Journal of Chromatography $A$, vol. 983, no. 1-2, pp. 35-42, 2003.

[25] H. Kataoka, "Derivatization reactions for the determination of amines by gas chromatography and their applications in environmental analysis," Journal of Chromatography A, vol. 733, no. 1-2, pp. 19-34, 1996.

[26] D. G. I. Kingston, J. T. Bursey, and M. M. Bursey, "Intramolecular hydrogen transfer in mass spectra. II. The McLafferty rearrangement and related reactions," Chemical Reviews, vol. 74, no. 2, pp. 215-242, 1974.
[27] K. Zaitsu, M. Katagi, H. T. Kamata, A. Miki, and H. Tsuchihashi, "Discrimination and identification of regioisomeric $\beta$ keto analogues of 3,4-methylenedioxyamphetamines by gas chromatography-mass spectrometry," Forensic Toxicology, vol. 26, no. 2, pp. 45-51, 2008. 


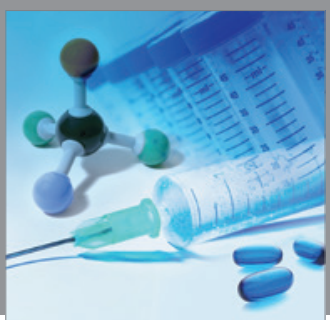

International Journal of

Medicinal Chemistry

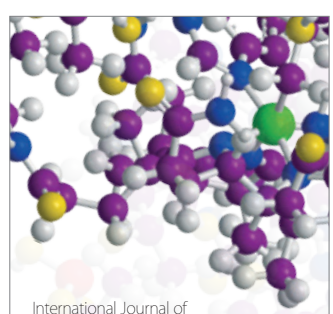

Carbohydrate Chemistry

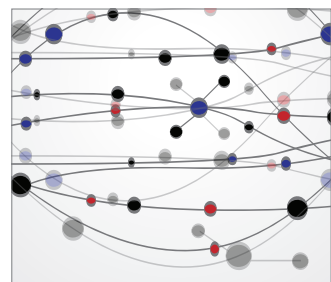

The Scientific World Journal
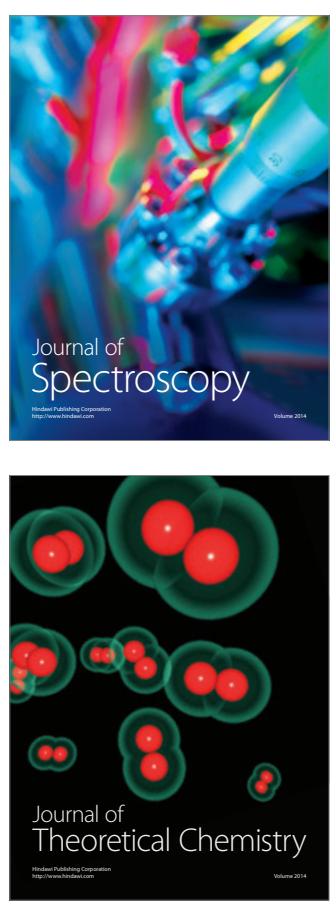
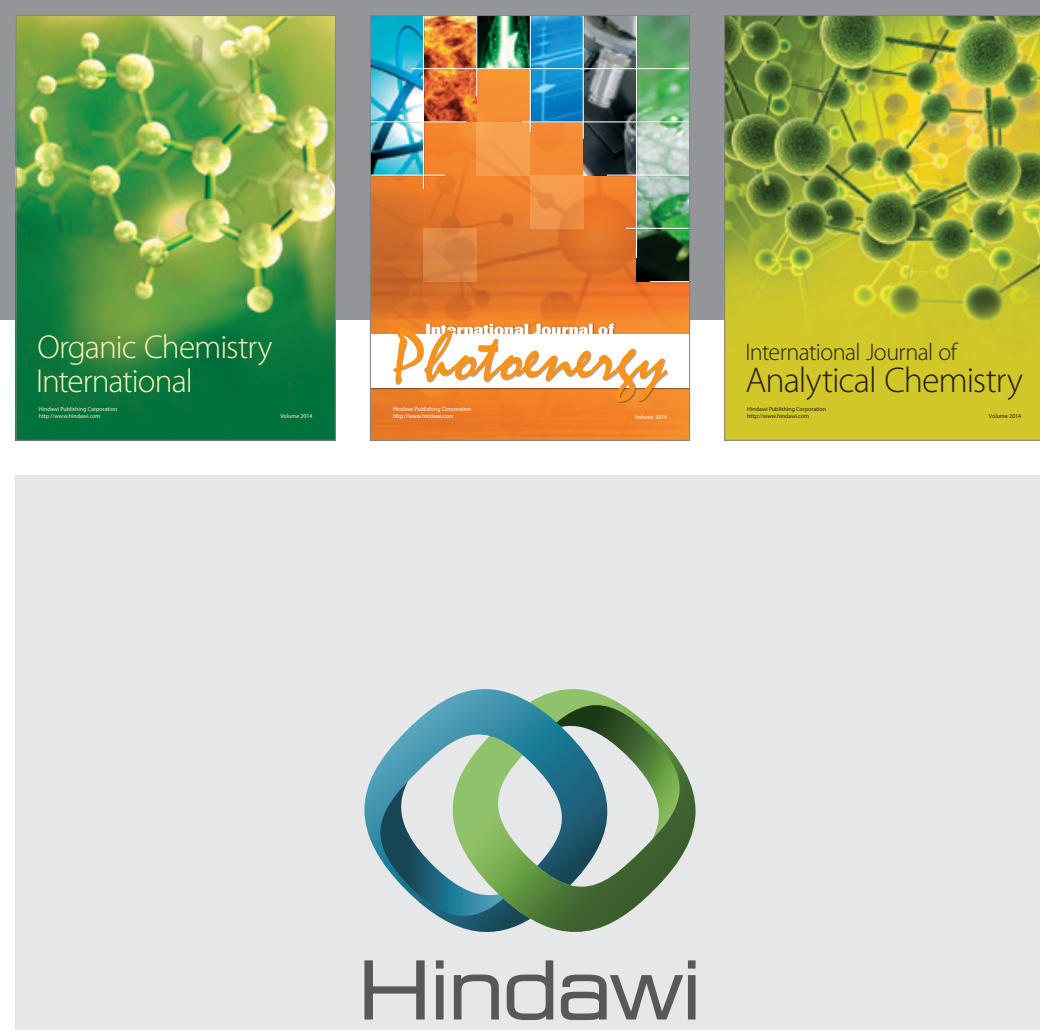

Submit your manuscripts at

http://www.hindawi.com
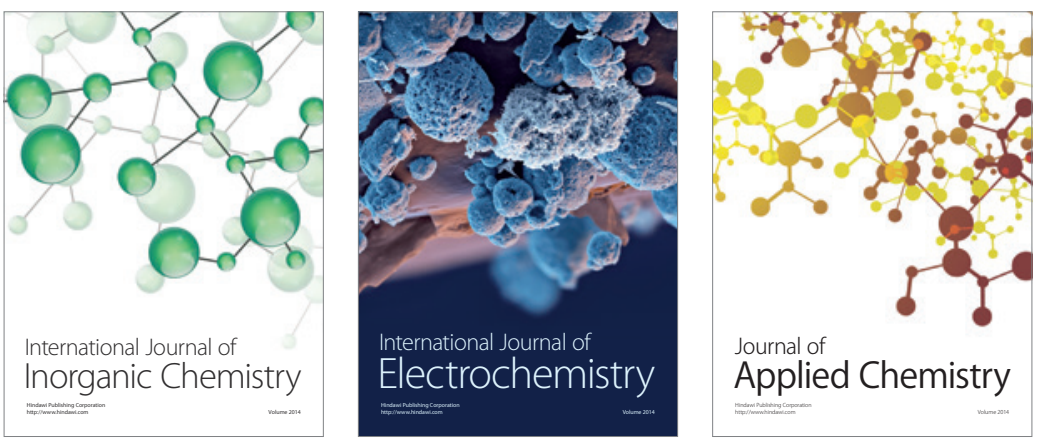

Journal of

Applied Chemistry
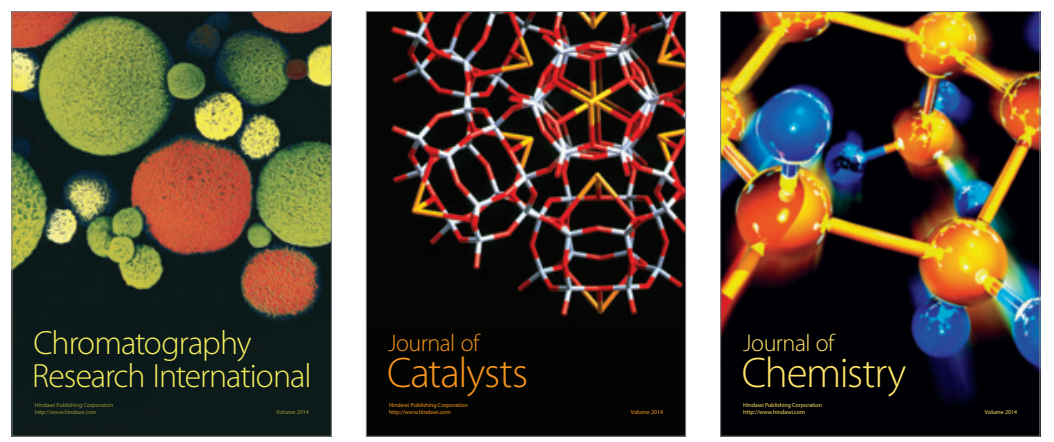
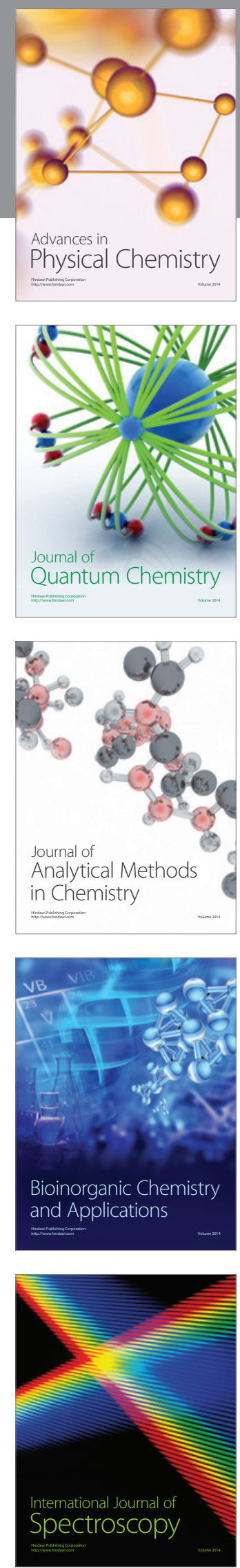